\title{
Comparing transmission reconstruction models with Mycobacterium tuberculosis whole genome sequence data
}

Benjamin Sobkowiak ${ }^{1,2, \ddagger^{*}}$, Kamila Romanowski ${ }^{2,3}$, Inna Sekirov ${ }^{2,4}$, Jennifer L Gardy ${ }^{5}$, James Johnston ${ }^{1,2}$

1. Division of Respiratory Medicine, University of British Columbia, Vancouver, Canada.

2. British Columbia Centre for Disease Control, Vancouver, Canada.

3. School of Population and Public Health, University of British Columbia, Vancouver, Canada.

4. Department of Pathology and Laboratory Medicine, University of British Columbia, Vancouver, BC, Canada.

5. Bill and Melinda Gates Foundation, Seattle, WA, USA.

‡. Present address. Department of Mathematics, Simon Fraser University, Burnaby, Canada

*Correspondence: Benjamin_sobkowiak@sfu.ca

Keywords: Tuberculosis, transmission, network reconstruction, bioinformatics, Bayesian inference. 


\section{Abstract}

Pathogen genomic epidemiology is now routinely used worldwide to interrogate infectious disease dynamics. Multiple computational tools that reconstruct transmission networks by coupling genomic data with epidemiological modelling have been developed. The resulting inferences are often used to inform outbreak investigations, yet to date, the performance of these transmission reconstruction tools has not been compared specifically for tuberculosis, a disease process with complex epidemiology that includes variable latency periods and within-host heterogeneity. Here, we carried out a systematic comparison of seven publicly available transmission reconstruction tools, evaluating their accuracy in predicting transmission events in both simulated and real-world Mycobacterium tuberculosis outbreaks. No tool was able to fully resolve transmission networks, though both the single-tree and multi-tree input implementations of TransPhylo identified the most epidemiologically supported transmission events and the fewest false positive links. We observed a high degree of variability in the transmission networks inferred by each approach. Our findings may inform an enduser's choice of tools in future tuberculosis transmission analyses and underscore the need for caution when interpreting transmission networks produced using probabilistic approaches. 


\section{Introduction}

2 Tuberculosis (TB), predominately caused by infection with Mycobacterium tuberculosis

3 (Mtb), remains a major global public health concern, with an estimated 10 million people

4 developing disease and 1.5 million deaths in $2020^{1}$. Accelerating TB burden reduction

5 to meet EndTB's elimination goals ${ }^{2}$ will require broad improvements in our

6 understanding of TB pathogenesis, along with improvements in access to point-of-care

7 diagnostics, prevention, and effective treatment. It will also require a deeper

8 understanding of Mtb transmission dynamics within different population settings -

9 information critical to managing local outbreaks and developing data-driven prevention

10 and control strategies based on an understanding of the clinical, social network, and

11 environmental factors that drive onward transmission.

At the individual and population levels, identifying linked cases and broader

14 transmission clusters has traditionally been done through a combination of DNA

15 fingerprinting techniques and field-based epidemiological methods, such as contact

16 tracing ${ }^{3}$. While this approach can be effective in well-resourced settings with limited TB

17 burden, it can be prohibitively labor-intensive in high transmission settings with limited

18 laboratory and field epidemiology capacity. Moreover, contact tracing relies on the self-

19 reporting of contacts ${ }^{4}$ and is therefore inherently subjective and reliant on interviewee

20 recall, which can be particularly problematic for an illness with a prolonged infectious

21 period, such as TB; this can be limiting to the epidemiologists' ability to accurately

22 reconstruct full transmission histories. 
24 Recently, Mtb whole genome sequencing (WGS) data has enabled finer-scale

25 resolution of Mtb transmission events, although relating whole genome variation to

26 direct transmission events can still be complex, particularly when clinical and

27 epidemiological information, such as symptom onset date, bacillary load, and known

28 contacts and places of social aggregation, are incomplete or unavailable. The most

29 simplistic method is to set a threshold for the maximum level of genetic variation

30 permitted to define transmission between samples, often using a single nucleotide

31 polymorphism (SNP) threshold. This technique has been applied successfully in

multiple settings to draw insights into transmission dynamics ${ }^{5,6}$, but it often lacks

sufficient resolution to determine the direction and timing of individual transmission

events $^{7,8}$, and is further complicated by the lack of consensus on SNP thresholds ${ }^{5,9-11 .}$

A more sophisticated approach to transmission reconstruction using genomic data relies

37 on phylogenetic trees. This method, phylodynamics ${ }^{12}$, can characterize macro-scale network patterns ${ }^{13}$ and estimate transmission events and timing for some pathogens, such as RNA viruses ${ }^{14}$. However, in many bacterial pathogens, including Mtb, multiple factors complicate the use of phylodynamic approaches. These can include within-host evolution driven by variable latency periods or chronic infection, variable mutation rates,

42 and mobile genetic elements ${ }^{14-16}$. Clinical sampling can also affect our interpretation of

43 genomic data - in cases where a host is infected with and capable of transmitting

44 multiple lineages of Mtb, a sputum sample may contain or preferentially culture only a

45 single lineage, leading to incorrect transmission inferences ${ }^{17,18}$. 
47 Recently, several groups have developed computational tools that combine genomic variation with an underlying epidemiological model (e.g. susceptible-infected-recovered (SIR) ${ }^{8,19}$ or stochastic branching process ${ }^{16,20}$ to estimate the probability of individual-

50 level transmission events from genomic data alone. These tools take either a multi-

51 sequence alignment or phylogenetic trees as input, along with other information, such

52 as sampling date, and mainly employ a Bayesian Markov Chain Monte Carlo (MCMC)

53 framework to account for the high dimensionality and computational complexity of the

54 resulting models, as well as incorporating other epidemiologically derived parameters.

Many of these tools have been used in Mtb transmission studies ${ }^{21-23 ;}$ however, their performance on Mtb data has not yet been systematically compared. In this study, we evaluated seven model-based transmission inference approaches; seqTrack ${ }^{24}$,

59 TransPhylo single tree input ${ }^{16}$, TransPhylo multiple tree input ${ }^{25}$, Outbreaker2 ${ }^{26}$, Phybreak ${ }^{20}$, SCOTTI ${ }^{27}$, and BEASTLIER ${ }^{19}$. We first simulated ten Mtb outbreaks and

61 the resulting genomic data and evaluated the accuracy of each method in identifying

62 known in silico transmission links. Next, we ran each tool on real-world WGS data from

63 clinical Mtb isolates collected in British Columbia, Canada, (BC) between 2005-2014.

64 We compared the resulting high-probability transmission events inferred by each

65 approach to known epidemiologically linked case-contact pairs and examined the

66 methods' different posterior estimates of key transmission parameters. Our results

67 represent the first evaluation and comparison of multiple tools for reconstructing Mtb 68 transmission networks using genomic data. 


\section{Materials and methods}

\section{Clinical sample information}

72 Study data were collected in BC, a low TB burden region with a population of 5 million

73 people and a TB incidence of 6 cases per 100,000 population ${ }^{28,29}$. Mtb samples were

74 obtained from the Public Health Laboratory (PHL) of the BC Centre for Disease Control

75 (BCCDC), which maintains an archive of all Mtb culture-positive isolates from

76 individuals diagnosed with TB in the province. From 2,915 culture-positive TB cases

77 diagnosed between 2005-2014, genomic DNA was extracted from 2,290 isolates, one

78 sequence per person, and analysed using MIRU-VNTR genotyping. Sample

79 preparation, DNA extraction, and genotyping methods are described elsewhere ${ }^{30}$.

80 Ethics were obtained from the University of British Columbia (certificate H12-00910).

81

\section{Clinical sample sequence analysis}

83 Whole genome sequencing was performed at the BC Genome Sciences Centre on the

84 Illumina HiSeq platform on all isolates with a shared MIRU-VNTR genotyping pattern.

85 This resulted in 1,014 high-quality whole genome Mtb sequences of 125bp paired-end

86 reads, with an average depth of coverage of $>100 x$. Sequencing reads were quality

87 checked using the FastQC tool and processed to remove adapter sequences and low quality reads using Trimmomatic ${ }^{31}$. Reads were mapped to the H37Rv reference strain

89 (Genbank no.: NC_000962) using BWA mem ${ }^{32}$.

91 Variant calling was conducted using the GATK suite of programs ${ }^{33}$, including

92 HaplotypeCaller and GenetypeGVCFs, with SNPs used in the subsequent analysis. 
93 Low confidence variants (phred quality score $Q<20$, read depth $D P<5$ ) and sites with a

94 missing call (either through non-mapping of sequencing reads or low coverage) in $>10 \%$

95 of isolates were removed. Heterozygous sites were called as the reference or

96 alternative allele where $\geq 80 \%$ of mapped reads corresponded at these positions;

97 otherwise, these sites were assigned as an ambiguous call 'N'. Variants in repetitive

98 regions, PE/PPE genes and at known resistance-conferring genes were also removed

99 from the transmission analysis.

100

101 Putative transmission clusters were identified by grouping isolates with a shared MIRU-

102 VNTR pattern, with preliminary analysis revealing that one cluster of 65 individuals,

103 MCLUST012, was comprised of two distinct sub-clusters of 49 and 16 individuals and

104 thus was split for further analysis. We tested transmission reconstruction tools on the

105 resulting 12 clusters that we categorized as either small (four clusters between 5 and 9

106 isolates) or large (eight clusters with $\geq 10$ isolates) and for which contract tracing data

107 was present for at least one epidemiological linked pair of cases in the cluster.

\section{Phylogenetic methods}

110 Timed phylogenies were produced for each MIRU-VNTR cluster separately with either

111 BEAST v1.10.4 ${ }^{34}$ (for BEASTLIER) or BEAST2 v2.6.3 ${ }^{35}$ (for TransPhylo), calibrated at

112 the tips by date of collection. A multiple sequence alignment of concatenated SNPs was

113 used as input, with XML files modified manually to estimate the number of invariant

114 sites. An appropriate substitution rate model was chosen for each cluster by producing

115 a maximum likelihood tree using IQTREE ${ }^{36}$ with 1000 bootstraps and applying the 
116 'ModelFinder' algorithm ${ }^{37}$ to find the model with the lowest Bayesian information

117 criterion (BIC) score. We assessed the temporal signal in each cluster (the correlation

118 between collection date and genomic distance) using TempEst ${ }^{38}$. Where some

119 temporal signal was found $\left(R^{2}>0.1\right)$, a strict molecular clock was used, otherwise we

120 used a relaxed lognormal clock prior and supplied both models with an initial prior of

$1211 \times 10^{-7}$ with a lognormal distribution, updated through MCMC iterations. Tree building

122 parameters were further optimised for each cluster by conducting separate preliminary

123 runs of $10^{8} \mathrm{MCMC}$ iterations whilst varying the population model (constant, exponential, 124 and Bayesian Skyline). Results were assessed using the posterior marginal likelihood

125 estimates and run convergence in Tracer $\mathrm{v} 1.7 .1^{39}$, discarding the $10 \%$ of trees as the

126 burn-in. The optimal tree building parameters chosen for each cluster are detailed in the

127 supplementary materials along with the posterior estimates of cluster-specific

128 substitution rate and tree height (Supplementary Table S1).

130 Final time-calibrated phylogenies were produced from BEAST2 runs of $10^{9} \mathrm{MCMC}$

131 iterations with the optimised prior parameters, sampling every $10,000^{\text {th }}$ tree and

132 discarding the first $10 \%$ of trees as the burn-in. Where a single phylogenetic tree was

133 used as input for reconstruction models, a maximum clade consensus tree was

134 obtained using Tree Annotator v2.6.3 ${ }^{35}$ with median node heights. For analysis of

135 multiple phylogenetic trees simultaneously with TransPhylo, a random selection of 50

136 trees was drawn from the posterior selection of 10,000 trees, discarding the $50 \%$ burn-

137 in, using a custom bash script. 


\section{Transmission network reconstruction models}

140 We tested six tools for reconstructing transmission networks on the simulated outbreaks

141 and real dataset of clinically derived Mtb isolates: seqTrack ${ }^{24}$, TransPhylo ${ }^{16}$,

142 Outbreaker2 ${ }^{26}$, Phybreak ${ }^{20}$, SCOTTI ${ }^{27}$, and BEASTLIER ${ }^{19}$. There has been a recent

143 extension to TransPhylo that allows for inference across multiple input trees sharing

144 parameter estimates to account for uncertainty in the tree building process ${ }^{25}$ - this was

145 also tested and is referred to as TransPhyloMT. All approaches utilize genomic data,

146 either directly as a multiple sequence alignment, or indirectly from a timed phylogenetic

147 tree, as well as sampling dates. Input data types and epidemiological characteristics of

148 the tested methods are shown in Table 1, along with specific model features, such as

149 accounting for non-sampled hosts and within-host evolution.

151 All tools allow specification of a prior estimate of either the generation time or host

152 infectiousness interval to model the time interval between hosts becoming infected. For

153 these measures, a long-tailed gamma distribution with parameters of shape $=1.3$ and

154 scale $=3.33$ was chosen, previously used in transmission analysis of this TB population

155 and in other settings with effective active case-finding strategies ${ }^{16,40}$. Some methods

156 also allow for a prior estimate of the sampling distribution - the time between a host

157 becoming infected and the sample being taken; for these, we set a gamma distribution

158 with shape and scale parameters of 1.1 and $2.5^{16,40}$. All methods, except BEASTLIER

159 and seqTrack, allow for incomplete sampling by including unobserved hosts in predicted

160 transmission networks (Table 1). Where permitted, we set a prior sampling proportion of 161 around $80 \%$ complete sampling of TB cases in BC, a well-resourced setting with good 
162 active case finding ${ }^{41}$. Full details for the model-specific inputs and prior parameter

163 estimates can be found in the Supplementary Materials.

Model performance on clinical data from British Columbia

166 In the absence of a gold-standard for confirmed transmission, the performance of each

167 approach on clinical Mtb data was evaluated by calculating model sensitivity and

168 positive predictive value (PPV) using the number of epidemiologically linked case-

169 contact pairs that were correctly identified (Supplementary Tables S2 and S3). Case-

170 contact pairs were defined as per ${ }^{41}$ and included when both isolates are present in the

171 same MIRU-VNTR defined cluster. Correct predictions (true positives) were classified

172 as an inferred transmission event between a known case-contact pair identified with a

173 probability of $\geq 0.5$, with sensitivity defined as the proportion of the total case-contact

174 pairs correctly identified for each cluster. Where multiple donor hosts were identified for

175 a recipient strain with a probability of $\geq 0.5$, only the highest probability link was

176 considered. While not all true transmission events were captured in the case-contact

177 pairs, meaning some true positive links will be misclassified as false positives, we

178 attempted to account for models simply identifying a greater number of true

179 transmission events through inferring a high number of links overall, including false

180 positives, by calculating the PPV of each method. The PPV was calculated as the

181 proportion of correct predictions in the total number of transmission links $(P \geq 0.5)$

182 inferred per cluster. 
184 Additionally, we compared posterior estimates of transmission parameters to evaluate

185 the credibility of the links predicted by each approach. Firstly, previous work has

186 reported that a signal of direct transmission in Mtb is low divergence between

187 sequences, with most transmission pairs differing by $0-5$ SNPs ${ }^{5,7,42}$. Therefore, while

188 there may be some cases of sequences differing by more than 5 SNPs in direct recent 189 transmission events, we can work under the assumption that most transmission will be

190 between isolates with few SNP differences and an abundance of links between highly

191 diverged sequences may indicate erroneous inferences. Five of the tested approaches,

192 excluding seqTrack and SCOTTI, inferred an infection time for each host and thus we

193 were able to calculate transmission intervals in these models, defined as the time

194 between donor and recipient host infection. While this interval can be highly variable in

195 TB, evidence suggests that most secondary transmission events occur within the two

196 years after infection ${ }^{41,43}$ and this should be reflected in the inferred transmission

197 networks.

198

199 Simulated Mtb transmission clusters

200 We simulated ten transmission clusters reflecting the epidemiology and sampling

201 strategy in the clinical Mtb clusters used in this study using the 'sim.phybreak' function

202 in Phybreak ${ }^{20}$. Transmission clusters were simulated with an observed host number of

20350 , which we then sampled 6 years either side of the median sampling date to simulate

204 an ongoing outbreak that was active before the date of the first observed host. To allow

205 for the presence of non-sampled hosts within clusters, we randomly down-sampled the 
206 observed hosts within each cluster by $20 \%$ to simulate incomplete sampling, which

207 resulted in a final sample number in clusters of between 28 and 40 observed hosts.

208

209 Epidemiological parameters for the simulated clusters were chosen to reflect clinical

210 Mtb clusters, thus we chose the same gamma generation and sampling time

211 distributions as described previously. The sequence length was set as length of the

212 H37Rv Mtb reference stain at 4.4M base pairs and we allowed for within-host evolution

213 at the rate of 1.48 as previously estimated $16,40,44$ and a mutation rate of 0.5 SNPs per

214 genome per year, taken from estimates in our clinical data using BEAST2 v2.6.3 ${ }^{35}$,

215 which is in line with previous estimates of the Mtb mutation rate ${ }^{45}$. The simulator takes

216 these epidemiological estimates and produces simulated sequence data and

217 transmission networks, including the time and direction of transmission.

219 Simulated genome sequences and sampling dates of the observed hosts were used as

220 input for each tested approach, with the resulting predicted transmission events

221 compared against known transmission to evaluate model sensitivity (the proportion of

222 the true transmission links correctly identified) and PPV (the correct transmission links

223 as a proportion of the total inferred transmission links (true positives + false positives)).

224 Additionally, simulated transmission networks included information on who-infected-

225 whom and so sensitivity and PPV for inferring the correct direction of transmission was

226 also calculated for each model.

227

228 Results 


\section{Simulated transmission clusters}

230 We first assessed the performance of the described methods by identifying the number

231 of transmission events correctly predicted in ten simulated Mtb outbreaks. Figure 1A

232 shows the sensitivity and PPV of each approach for all simulated clusters for

233 transmission links with a probability of $\geq 0.5$, irrespective of the direction of transmission

234 (i.e., if host $\mathrm{i}$ transmitted to host $\mathrm{j}$ in the true transmission network, a prediction of $\mathrm{i}->\mathrm{j}$ or

235 j -> i would be scored as correct). Outbreaker2 correctly predicted the highest proportion

236 of the true transmission events with a median sensitivity of 0.5 (IQR $0.37-0.58$ ),

237 followed by TransPhylo (sensitivity 0.4 , IQR $0.35-0.44$ ), though Phybreak and

238 TransPhyloMT achieved comparable scores (median 0.4, IQR $0.36-0.49$ and median

2390.38 , IQR $0.35-0.43$ respectively). BEASTLIER, seqTrack and SCOTTI performed

240 poorly in predicting transmission in the simulated clusters and, notably, SCOTTI did not

241 infer any transmission links with a probability of $\geq 0.5$ in any cluster (Supplementary

242 data, Table S2). There were marked differences in the PPV of each approach when

243 predicting correct transmission links in simulated clusters, with TransPhyloMT achieving

244 the highest PPV (median 0.68, IQR $0.62-0.72$ ), followed by TransPhylo (median 0.58,

245 IQR $0.48-0.66)$. The high PPV of TransPhyloMT compared to the other higher

246 sensitivity approaches was due to fewer false positive transmission links predicted with

247 a probability of $\geq 0.5$ (Supplementary data, Table S2).

249 All models predicted fewer correct transmission links when the direction of transmission

250 was considered (i.e., i -> j $\neq$ j -> i) (Figure 1B). Phybreak achieved the highest

251 sensitivity, predicting around a third of true directional transmission links (median 0.33, 
252 IQR $0.27-0.42$ ), followed by Outbreaker2 (median 0.32, IQR $0.3-0.36$ ) and both

253 TransPhylo and TransPhyloMT (median 0.26, IQR $0.24-0.31$, and median 0.26, IQR

$2540.25-0.33)$. The PPV of TransPhyloMT was again highest when identifying the

255 direction of transmission, with typically around half of the inferred transmission events

256 predicted using this approach being a confirmed link (median 0.5, IQR 0.41 - 0.54).

Clinical M. tuberculosis data from British Columbia

259 We next applied each method to real-world WGS data. We observed a high degree of

260 variation in transmission networks inferred by each method, reflecting the different

261 underlying model algorithms and parameters employed by each approach. An example

262 of the differences in consensus transmission networks is shown in Figure 2, which

263 illustrates transmission events inferred by two methods, Phybreak and TransPhylo, for

264 cluster MCLUST004 ( $\mathrm{N}=9$ cases).

265

266 Table 2 shows the PPV and sensitivity of each model for predicting transmission within

267 the clinical Mtb clusters, as both a total for all clusters and separated by small $(\mathrm{N}<10)$

268 and large $(\mathrm{N} \geq 10)$ clusters. Overall, the highest number of the epidemiologically linked

269 case-contact pairs were identified with TransPhylo (20/120; sensitivity 17\%) followed by

270 TransPhyloMT (18/120; sensitivity 15\%). SCOTTI again performed poorly, predicting

271 just a single case-contact pair in the resulting transmission links (sensitivity 1\%). There

272 were clear differences between model sensitivity in the large and small clusters, with the

273 best scoring model, Phybreak, identifying just 16/106 (15\%) case-contact pairs in large

274 clusters, while TransPhyloMT correctly identified 6/14 (43\%) case-contact pairs in the 
275 small clusters. PPV was also highest using TransPhyloMT for all clusters, as well as

276 when considering small and large clusters separately, with 18/63 (29\%)

277 epidemiologically linked case-contact pairs inferred as high-confidence transmission

278 links, suggesting this method will infer the lowest number of false positive transmission

279 events.

280

281 Closer inspection revealed that all models predicted a low proportion of the case-

282 contact pairs in the largest two clusters in the population, MCLUST001 and

283 MCLUST002 (Supplementary Table S3), this though may be due to the complicated

284 epidemiology in these two large outbreaks ${ }^{46}$ and so we can be less confident of the

285 validity of the case-contact pairs. Indeed, removing these two clusters from the analysis

286 significantly increased the sensitivity scores of most models, with Phybreak now

287 correctly predicting 13/33 (39\%) of case-contact pairs in the remaining large clusters.

Posterior estimates of SNP distance and transmission interval between hosts in

predicted direct transmission events revealed differences between tested approaches.

291 The median SNP distance in high probability transmission links between observed hosts

292 was low for all models, ranging from 0 SNPs (IQR 0 - 0 SNPs) in seqTrack, which only

293 predicted transmission events between identical sequences, to 5 SNPs (IQR 2 - 9

294 SNPs) for BEASTLIER (Figure 3A). BEASTLIER and Phybreak also predicted several

295 transmission events between relatively divergent isolates (> 30 SNP differences), which

296 may be due to these models not allowing for non-sampled hosts, resulting in incorrect

297 direct links between hosts when there are many unobserved cases in the real 
298 transmission chain. Figure 3B illustrates the differences in transmission interval

299 estimated between observed hosts in direct transmission events for models that inferred

300 infection times. We can see that all tested approaches estimate that the majority of

301 secondary cases are within the first two years after donor host infection (BEASTLIER

302 median 0.2 years, IQR $0.2-0.5$, to Phybreak median 1.1 years, IQR $0.5-2.1$ ).

303 Phybreak and BEASTLIER predict some high-probability transmission events between

304 hosts where the infection time is many years, with a maximum value of 8.8 years

305 between host infection times using BEASTLIER. While long periods between

306 transmission events have been observed in Mtb due to latency of disease onset, the

307 links inferred here are not supported by epidemiological evidence and as these only

308 occur in models only allowing transmission between observed hosts, these are likely an

309 artifact of the model assumptions.

\section{Sensitivity analysis}

312 To assess the impact of the setting the accepted probability threshold of transmission

313 links at $P \geq 0.5$ in the main analysis, we re-calculated the sensitivity and PPV of each

314 approach for the clinical BC MIRU-VNTR clusters at a higher and lower probability

315 threshold of $P \geq 0.75$ and $P \geq 0.25$. As expected, we found an increase in the sensitivity

316 of most methods when decreasing the probability threshold to $\geq 0.25$, and a decrease in

317 sensitivity at $P \geq 0.75$ (Supplementary figure S1). These differences were most

318 pronounced in Phybreak, TransPhylo, and TransPhyloMT, where a lower probability

319 threshold allowed for a greater number of case-contact pairs to be identified. Lowering

320 the probability threshold also increased the overall number of accepted transmission 
321 links with each tested model, which may also increase the number of false positive

322 calls. The PPV of both TransPhylo and TransPhyloMT decreased markedly at a

323 probability threshold of $\geq 0.25$ compared to $\geq 0.5$, with a greater number of false positive

324 transmission links identified (Supplementary figure S2). The PPV of seqTrack and

325 Outbreaker2 increased at $P \geq 0.25$ compared to $P \geq 0.5$, though the relative difference

326 between these values was small. Interestingly, we saw a decrease in PPV for all tools at

327 the $\geq 0.75$ probability threshold compared to $\geq 0.5$ as the reduction in the number true

328 positive calls was greater than the decrease in false positives at this higher probability

329 threshold.

331 Discussion

332 We present the first systematic comparison of the available methods for reconstructing

333 TB transmission networks using whole genome sequence data, using input data from

334 both simulated outbreaks and real-world Mtb transmission clusters. Our results

335 demonstrate that the choice of analytical tool can impact the structure and accuracy of

336 predicted transmission events. While it is difficult to infer full Mtb transmission networks

337 using only genomic and temporal data owing to the low diversity characteristic of TB

338 outbreaks, we have shown certain models performed markedly better than others in

339 both real-world and simulated data. As such, the results presented here can help to

340 guide the choice of methodology for Mtb transmission investigations.

342 In simulated outbreaks, Outbreaker2 predicted the highest number of 'true' transmission

343 events, with an average of half the links between hosts in each cluster correctly 
344 identified, and a third predicted with the correct direction of transmission. Phybreak,

345 TransPhylo and TransPhyloMT achieved comparable results for identifying known

346 transmission between hosts, though the overall number of high probability links inferred

347 by Outbreaker2 and Phybreak was higher than with TransPhylo and TransPhyloMT.

348 This greatly increased the number of false positive transmission events predicted by

349 both Outbreaker2 and Phybreak, which resulted in the PPV of these tools being

350 significantly lower than TransPhylo and TransPhyloMT. This is also seen in the

351 specificity of each approach on the simulated clusters; while the differences are not as

352 large, TransPhylo and TransPhyloMT both outperformed Phybreak and Outbreaker2

353 (Supplementary Figure S3). Therefore, these results indicate that TransPhylo,

354 particularly TransPhyloMT, may be the most effective tool for identifying transmission

355 events between sampled hosts in a population, while reducing the number of spurious

356 links between hosts that are wrongly predicted with a high probability.

A limitation in this study was an absence of a gold-standard for transmission within the

359 clinical TB population. We were able to provide strong evidence of 'correct' links by

360 including several epidemiologically linked case-contact pairs in our analysis, but these

361 data were drawn from contact tracing questionnaires where participants may not

362 produce a complete network of potential sources of infection. Nonetheless, we

363 hypothesized that the presence of epidemiological and genomic linkage between hosts

364 increased the likelihood of these links being true transmission events and thus these

365 links were used as a measure of 'ground-truth' in analysis of the clinical TB data. 
367 Unfortunately, the ability of all tested models to identify case-contact pairs was relatively

368 low, with TransPhylo performing best but only finding $17 \%$ of epidemiologically

369 supported links. The poor performance of models appears to be driven by the low

370 number of case-contact pairs inferred in the largest two clinical transmission clusters,

371 MCLUST001 and MCLUST002. These clusters were part of two separate TB outbreaks

372 with complex demography, that included people experiencing homelessness and poly-

373 substance use ${ }^{47,48}$. The disparity between the inferred transmission networks and the

374 reported case-contact information may be due to difficulty in contact tracing in these

375 settings, decreasing the likelihood of the contact data containing the true transmission

376 links. Indeed, after removing MCLUST001 and MCLUST002 from analysis, the overall

377 performance of most tools increased, with Phybreak identifying 39\% and TransPhylo

$37833 \%$ of the remaining epidemiologically linked case-contact pairs (Supplementary

379 Table S3).

381 In this study, TransPhyloMT achieved the highest PPV in clinical data, with epidemiologically linked case-contact pairs constituting $29 \%$ of the total high probability

383 transmission events inferred, rising to $55 \%$ in small clusters. In contrast, we found the 384 number of transmission events inferred by SCOTTI in the clinical Mtb data to be 385 particularly low, with only four direct transmission links inferred across all 12 clusters, 386 and only a single epidemiologically confirmed case-contact pair detected. The reason 387 for this may be two-fold; SCOTTI was not primarily developed to model transmission in 388 Mtb populations that are often characterised by few clonal lineages and low genomic 389 variation. In addition, we used a probability threshold for an accepted transmission link 
390 of $\geq 0.5$, while the majority of the reported links for real-life outbreaks tested in the

391 original publication of the model were below this value ${ }^{27}$. Indeed, when we decreased

392 the threshold value to $\geq 0.25$, we observed a vast increase in both the overall number of

393 predicted transmission events between sampled hosts (54 at $\geq 0.25$ compared to 4 at $P$

$394 \geq 0.5$ ), as well as an increase in the number of identified epidemiologically linked pairs

395 (7/120 at $\geq 0.25$ compared to $1 / 120$ at $P \geq 0.5)$, (Supplementary table S4). However,

396 even at this lower threshold, there were fewer epidemiologically linked pairs predicted

397 with SCOTTI when compared to other models at higher accepted probability threshold.

399 No single transmission network was fully resolved with any tested model; Phybreak

400 identified the highest proportion of known transmission events in a single cluster, while

401 TransPhyloMT achieved the highest PPV within a cluster. To increase the number of

402 correctly inferred transmission events, the threshold to accept an inferred link between

403 hosts could be reduced, but this will lead to a greater number of false positive links.

404 Previous studies have used these inference tools to gain insights in the Mtb

405 transmission dynamics notably identifying individual transmission events and increased

406 transmissibility within populations (e.g., ${ }^{21,23,25}$ ), by reconstructing transmission histories

407 based on accepting or rejecting links at a set probability threshold. We have

408 demonstrated that there can be doubt in the inferred transmission links, even with the

409 best performing models, and as such it may be valuable to provide the probability of

410 linkage between hosts, rather than a binary classifier probability threshold for accepting

411 transmission. 
413 As computational models for transmission inference are being improved to better reflect

414 the characteristics of disease outbreaks and incorporate more realistic epidemiological

415 parameters, we suggest that a TB-focused methodology will likely deliver more accurate

416 transmission networks than tools calibrated to be applicable across a range of pathogen

417 genomes. This is reflected in the results shown here, where the best performing

418 models, Phybreak, Outbreaker2, and TransPhylo, have been developed to account for

419 the complex epidemiology of infectious diseases like tuberculosis, including handling

420 within-host evolution and non-complete outbreak sampling ${ }^{16,20,26}$. Furthermore, some

421 modifications could be implemented to existing tools to improve the accuracy of inferred

422 transmission networks. For example, other forms of genomic variation could be used to

423 increase the observed variation between strains, such as small insertions and deletions

424 (INDELs) that are often found in single isolates ${ }^{49}$. This may improve resolution in

425 instances when multiple isolates are separated by very few SNPs, typical in highly

426 clonal Mtb populations. Additionally, Outbreaker2 can be supplied with custom prior

427 likelihood and movement functions to adapt the underlying model, as well as accepting

428 contact network data, and these modifications can be expected to improve the accuracy

429 of output networks when appropriately specified a priori.

431 Together with the performance of each model for predicting transmission events, a

432 further consideration when running these tools is the processing complexity and

433 runtime, which may influence the decision on the model to employ. This is particularly

434 true if the aim is to use these approaches for real-time surveillance of Mtb transmission

435 or in settings with limited computational resources. Whilst all the tested tools ran 
successfully using a desktop computer with a $2.6 \mathrm{GHz}$ processor and $16 \mathrm{~GB}$ memory, the

437 runtime of each approach varied considerably (Supplementary Table S5). The fastest

438 tool tested was seqTrack, with networks computed almost instantaneously for the

439 largest clinical Mtb cluster MCLUST002 ( $N=74$ cases). This is due to the simplicity of

440 this approach compared to other models, which employ a Bayesian framework that

441 must run over millions of MCMC iterations for chain convergence. However, this method

442 may not provide accurate transmission networks in populations with within-host

443 evolution such as Mtb and incomplete sampling, as shown by our results. While we

444 observed reasonable inference of transmission using both TransPhylo approaches,

445 these models took considerably longer to complete in cluster MCLUST002 ( 19 hours

446 for TransPhylo MT, including phylogeny construction) and this would need to be taken

447 into consideration when selecting this tool. These results only show limited comparisons

448 of the computational resources required by each approach and more work would be

449 required to test the impact of running fewer iterations on accuracy with different

450 computational infrastructure to properly benchmark each approach.

451

452 While this study is not an exhaustive analysis of all transmission network reconstruction

453 methodologies (e.g. ${ }^{50}$ ), we have presented results from selected models that have

454 been used for investigating pathogen transmission histories and where a software

455 package was freely available for implementation. In addition to the methods presented

456 here, we also attempted to reconstruct transmission using BadTrIP ${ }^{51}$, which also

457 employs Bayesian inference to infer transmission networks from sequence data and

458 accounts for site polymorphisms, transmission bottlenecks and sequencing error. This 
459 model, though, failed to reach convergence in five of the eight large clinical

460 clusters/sub-clusters of $\mathrm{BC}$ isolates when allowing to run for $5 \times 10^{8} \mathrm{MCMC}$ iterations,

461 and so was not included in this study.

462

463 In conclusion, we have systematically compared seven tools for reconstructing

464 transmission using genomic data to assess their utility for TB transmission analysis. The

465 results presented here indicate that the transmission networks produced by each

466 method can disagree markedly due to the differences in the underlying models. While

467 there are limitations in the accuracy of the transmission histories provided by all models,

468 we found that the multi-tree implementation of TransPhylo achieved the most effective

469 balance of identifying a relatively high number of true transmission links while predicting

470 fewer false positive events in both simulated and real-life clinical Mtb clusters. This

471 approach could be applied to gain some insights into Mtb transmission dynamics using

472 sequence data in settings with limited contact network information. These findings can

473 improve investigations into Mtb outbreaks and transmission dynamics and highlight

474 further areas of research to advance methods for transmission network reconstruction.

475

476

$477 \quad$ References

478 1. World Health Organization. Global tuberculosis report 2021. (Licence: CC BY-NC-

479 SA 3.0 IGO., 2021).

480 2. World Health Organization. Global tuberculosis report 2020. (2020).

$4813 . \quad$ World Health Organization. Recommendations for investigating contacts of 
persons with infectious tuberculosis in low- and middle-income countries. World Heal. Organ. 28-41 (2012).

484 4. Walker, T. M., Monk, P., Smith, E. G. \& Peto, T. E. A. Contact investigations for outbreaks of Mycobacterium tuberculosis: Advances through whole genome sequencing. Clin. Microbiol. Infect. 19, 796-802 (2013).

5. Walker, T. M. et al. Whole-genome sequencing to delineate Mycobacterium tuberculosis outbreaks: a retrospective observational study. The Lancet Infectious Diseases 13, 137-146 (2013).

6. Luo, T. et al. Whole-genome sequencing to detect recent transmission of Mycobacterium tuberculosis in settings with a high burden of tuberculosis. Tuberculosis 94, 434-440 (2014).

7. Stimson, J. et al. Beyond the SNP Threshold: Identifying Outbreak Clusters Using Inferred Transmissions. Mol. Biol. Evol. 36, 587-603 (2019).

8. Didelot, X., Gardy, J. \& Colijn, C. Bayesian inference of infectious disease transmission from whole-genome sequence data. Mol. Biol. Evol. 31, 1869-1879

9. Lee, R. S. et al. Reemergence and amplification of tuberculosis in the Canadian Arctic. J. Infect. Dis. 211, 1905-1914 (2015).

10. Yang, C. et al. Transmission of multidrug-resistant Mycobacterium tuberculosis in Shanghai, China: a retrospective observational study using whole-genome sequencing and epidemiological investigation. Lancet Infect. Dis. 17, 275-284 (2017).

504 11. Lieberman, T. D. et al. Genomic diversity in autopsy samples reveals within-host 
dissemination of HIV-associated Mycobacterium tuberculosis. Nat. Med. 22,

506 1470-1474 (2016).

507

12. Grenfell, B. T. et al. Unifying the Epidemiological and Evolutionary Dynamics of

Pathogens. Science (80-. ). 303, 327-332 (2004).

509

13. Colijn, C. \& Gardy, J. Phylogenetic tree shapes resolve disease transmission

patterns. Evol. Med. public Heal. 2014, 96-108 (2014).

511 14. Ypma, R. J. F., van Ballegooijen, W. M. \& Wallinga, J. Relating phylogenetic trees to transmission trees of infectious disease outbreaks. Genetics 195, 1055-62 (2013).

514 15. Romero-Severson, E., Skar, H., Bulla, I., Albert, J. \& Leitner, T. Timing and order of transmission events is not directly reflected in a pathogen phylogeny. Mol. Biol. Evol. 31, 2472-2482 (2014).

517 16. Didelot, X., Fraser, C., Gardy, J. \& Colijn, C. Genomic infectious disease epidemiology in partially sampled and ongoing outbreaks. Mol. Biol. Evol. 34, 997-1007 (2017).

17. Worby, C. J., Lipsitch, M. \& Hanage, W. P. Within-Host Bacterial Diversity Hinders

PLoS Comput. Biol. 10, (2014).

523 18. Didelot, X., Gardy, J. \& Colijn, C. Bayesian inference of infectious disease

526 19. Hall, M., Woolhouse, M. \& Rambaut, A. Epidemic Reconstruction in a 
PLoS Comput. Biol. 11, 1-36 (2015).

529 20. Klinkenberg, D., Backer, J. A., Didelot, X., Colijn, C. \& Wallinga, J. Simultaneous inference of phylogenetic and transmission trees in infectious disease outbreaks. PLoS Computational Biology 13, (2017).

532 21. Sobkowiak, B. et al. Bayesian reconstruction of Mycobacterium tuberculosis transmission networks in a high incidence area over two decades in Malawi reveals associated risk factors and genomic variants. Microb. Genomics 6, (2020).

22. Hatherell, H. A. et al. Declaring a tuberculosis outbreak over with genomic epidemiology. Microb. Genomics 2, 3-6 (2016).

538 23. Guerra-Assunção, J. A. et al. Large-scale whole genome sequencing of M. tuberculosis provides insights into transmission in a high prevalence area. Elife 2015, 1-17 (2015).

541 24. Jombart, T., Eggo, R. M., Dodd, P. J. \& Balloux, F. Reconstructing disease outbreaks from genetic data: A graph approach. Heredity (Edinb). 106, 383-390 (2011).

544 25. Xu, Y. et al. High-resolution mapping of tuberculosis transmission: Whole genome 545 sequencing and phylogenetic modelling of a cohort from Valencia Region, Spain. PLoS Med. 16, 1-20 (2019).

547 26. Campbell, F. et al. outbreaker2: A modular platform for outbreak reconstruction. BMC Bioinformatics 19, (2018).

549 27. De Maio, N., Wu, C. H. \& Wilson, D. J. SCOTTI: Efficient Reconstruction of 550 Transmission within Outbreaks with the Structured Coalescent. PLoS Comput. 
Biol. 12, 1-23 (2016).

552

553

554

555

556

557

558

559

560

561

562

563

564

565

566

567

568

569

570

571

572

573

28. BC Centre for Disease Control. TB in British Columbia: Annual Surveillance Report 2017. (2019).

29. LaFreniere, M., Hussain, H., He, N. \& McGuire, M. Tuberculosis in Canada: 2017. Canada Commun. Dis. Rep. 45, 68-74 (2019).

30. Guthrie, J. L. et al. Molecular Epidemiology of Tuberculosis in British Columbia, Canada: A 10-Year Retrospective Study. Clin. Infect. Dis. 66, 849-856 (2018).

31. Bolger, A. M., Lohse, M. \& Usadel, B. Trimmomatic: A flexible trimmer for Illumina sequence data. Bioinformatics 30, 2114-2120 (2014).

32. Li, H. \& Durbin, R. Fast and accurate short read alignment with Burrows-Wheeler transform. Bioinformatics 25, 1754-60 (2009).

33. McKenna, A. et al. The Genome Analysis Toolkit: A MapReduce framework for analyzing next-generation DNA sequencing data. Genome Res. 20, 1297-1303 (2010).

34. Drummond, A. J., Suchard, M. A., Xie, D. \& Rambaut, A. Bayesian phylogenetics with BEAUti and the BEAST 1.7. Mol. Biol. Evol. 29, 1969-1973 (2012).

35. Bouckaert, R. et al. BEAST 2.5: An advanced software platform for Bayesian evolutionary analysis. PLoS Comput. Biol. 15, 1-28 (2019).

36. Minh, B. Q. et al. IQ-TREE 2: New Models and Efficient Methods for Phylogenetic Inference in the Genomic Era. Mol. Biol. Evol. 37, 1530-1534 (2020).

37. Kalyaanamoorthy, S., Minh, B. Q., Wong, T. K. F., Von Haeseler, A. \& Jermiin, L. S. ModelFinder: Fast model selection for accurate phylogenetic estimates. Nat. Methods 14, 587-589 (2017). 
574 38. Rambaut, A., Lam, T. T., Carvalho, L. M. \& Pybus, O. G. Exploring the temporal structure of heterochronous sequences using TempEst (formerly Path-O-Gen). Virus Evol. 2, 1-7 (2016).

577 39. Rambaut, A., Drummond, A. J., Xie, D., Baele, G. \& Suchard, M. A. Posterior summarization in Bayesian phylogenetics using Tracer 1.7. Syst. Biol. 67, 901904 (2018).

40. Ayabina, D. et al. Genome-based transmission modeling separates imported tuberculosis from recent transmission within an immigrant population. 1-13 (2017). doi:10.1101/226662

41. Romanowski, K. et al. Using Whole Genome Sequencing to Determine the Timing of Secondary Tuberculosis in British Columbia, Canada. Clin. Infect. Dis. 50, 1052-1063 (2020).

42. Meehan, C. J. et al. Whole genome sequencing of Mycobacterium tuberculosis: current standards and open issues. Nat. Rev. Microbiol. 17, 533-545 (2019).

43. Ma, Y., Horsburgh, C. R., White, L. F. \& Jenkins, H. E. Quantifying TB transmission: a systematic review of reproduction number and serial interval estimates for tuberculosis. Epidemiol. Infect. 146, 1478-1494 (2018).

44. $\mathrm{Xu}, \mathrm{Y}$. et al. Transmission analysis of a large tuberculosis outbreak in London: a mathematical modelling study using genomic data. Microb. Genomics 6, (2020).

593 45. Ford, C. B. et al. Mycobacterium tuberculosis mutation rate estimates from different lineages predict substantial differences in the emergence of drugresistant tuberculosis. Nat. Genet. 45, 784-790 (2013).

596 46. Gardy, J. L. et al. Whole-Genome Sequencing and Social-Network Analysis of a 
Tuberculosis Outbreak. N. Engl. J. Med. 364, 730-739 (2011).

598 47. Cook, V. J. \& Elwood, R. K. The burden of tuberculosis in Aboriginal communities of BC. B. C. Med. J. 50, 219 (2008).

600 48. Cheng, J. M. et al. A clonal outbreak of tuberculosis in a homeless population in the interior of British Columbia, Canada, 2008-2015. Epidemiol. Infect. 143, 3220-

603 49. Coll, F. et al. A robust SNP barcode for typing Mycobacterium tuberculosis complex strains. Nat. Commun. 5, 4-8 (2014).

605

606

607

608

609

610

611 3226 (2015).

50. Lau, M. S. Y., Marion, G., Streftaris, G. \& Gibson, G. A Systematic Bayesian Integration of Epidemiological and Genetic Data. PLoS Comput. Biol. 11, 1-27 (2015).

51. De Maio, N., Worby, C. J., Wilson, D. J. \& Stoesser, N. Bayesian reconstruction of transmission within outbreaks using genomic variants. PLoS Comput. Biol. 14, 1$23(2018)$. 
Table 1. The transmission network reconstruction tools evaluated in this study, detailing the epidemiological features and input data type for each approach.

\begin{tabular}{|c|c|c|c|c|c|c|c|c|}
\hline $\begin{array}{l}\text { Transmission } \\
\text { reconstruction } \\
\text { model }\end{array}$ & $\begin{array}{l}\text { Input data } \\
\text { type }\end{array}$ & $\begin{array}{c}\text { Uses } \\
\text { generation } \\
\text { time } \\
\text { distribution }\end{array}$ & $\begin{array}{l}\text { Prior } \\
\text { mutation } \\
\text { rate } \\
\text { estimate }\end{array}$ & $\begin{array}{c}\text { Can use } \\
\text { spatial data }\end{array}$ & $\begin{array}{c}\text { Can use } \\
\text { contact- } \\
\text { tracing data }\end{array}$ & $\begin{array}{l}\text { Allows for } \\
\text { within-host } \\
\text { evolution }\end{array}$ & $\begin{array}{c}\text { Allows for } \\
\text { non-sampled } \\
\text { hosts }\end{array}$ & $\begin{array}{l}\text { Infers } \\
\text { infection } \\
\text { times }\end{array}$ \\
\hline BEASTLIER ${ }^{19}$ & $\begin{array}{l}\text { BEAST1 } \\
\text { XML file }\end{array}$ & Yes & Yes & Yes & No & Yes & No & Yes \\
\hline Outbreaker2 26 & $\begin{array}{c}\text { Multi-FASTA } \\
\text { SNP } \\
\text { alignment }\end{array}$ & Yes & Yes & No & Yes & Yes & Yes & Yes \\
\hline Phybreak 20 & $\begin{array}{c}\text { Multi-FASTA } \\
\text { SNP } \\
\text { alignment }\end{array}$ & Yes & Yes & No & No & Yes & No & Yes \\
\hline SCOTTI 27 & $\begin{array}{l}\text { BEAST2 } \\
\text { XML file }\end{array}$ & No & Yes & No & No & Yes & Yes & No \\
\hline TransPhylo ${ }^{16}$ & $\begin{array}{c}\text { Timed } \\
\text { phylogenetic } \\
\text { tree }\end{array}$ & Yes & Yes & No & No & Yes & Yes & Yes \\
\hline TransPhyloMT 25 & $\begin{array}{c}\text { Timed } \\
\text { phylogenetic } \\
\text { trees* }\end{array}$ & Yes & Yes & No & No & Yes & Yes & Yes \\
\hline
\end{tabular}


Figure 1. Boxplots showing the sensitivity (blue boxes) and positive predictive value (PPV) (red boxes) of each

transmission reconstruction model for predicting known transmission events with a probability of $\geq 0.5$ in ten simulated TB clusters.

A

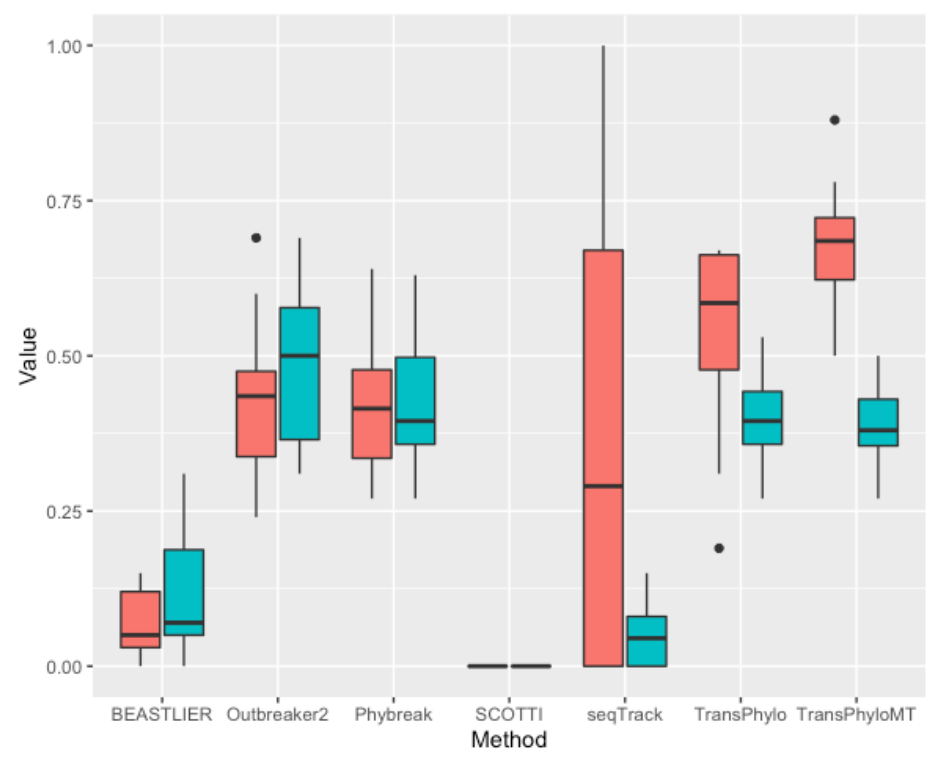

B

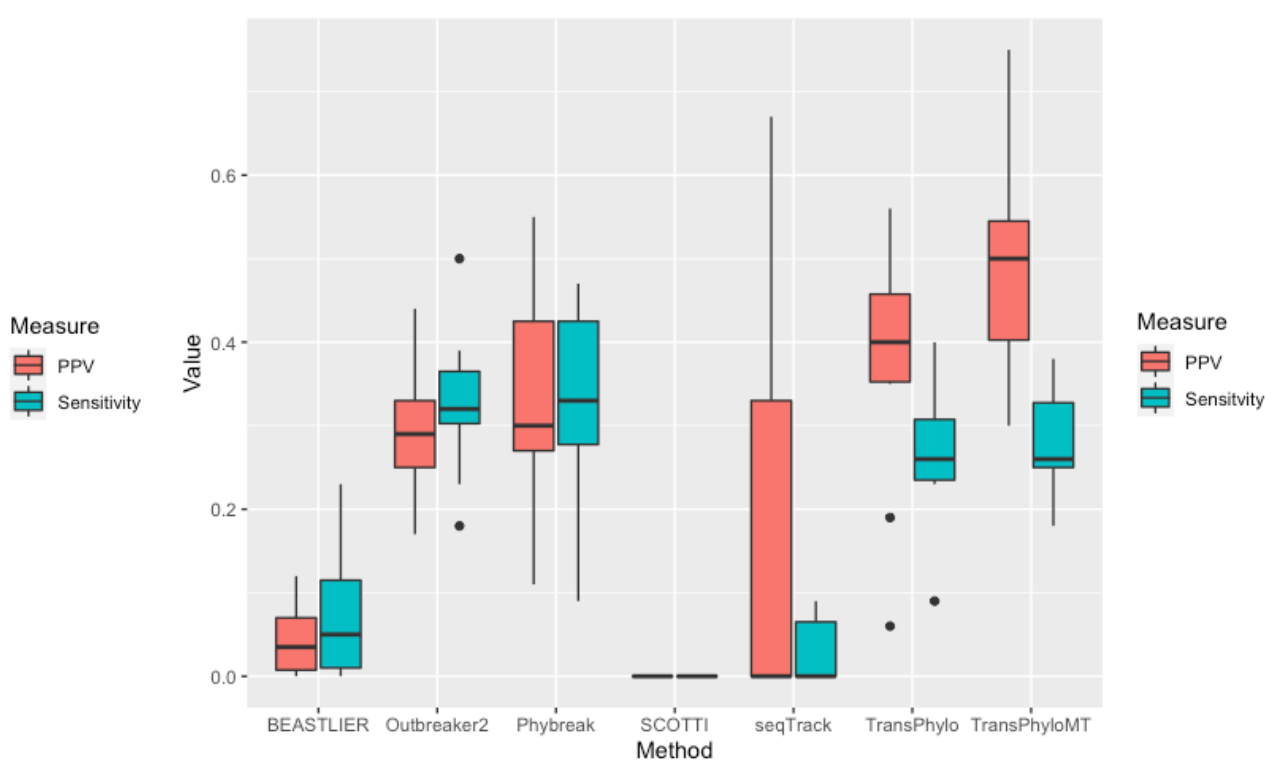


Figure 2. Example transmission networks inferred using two of the test methods, (A) Phybreak and (B) TransPhylo single tree, for the clinical BC transmission cluster, MCLUST004. Blue nodes represent the sampled hosts with sample names illustrated by letters A - I, black edges represent links with a posterior probability of $\geq 0.5$, and grey edges represent links < 0.5 , with the SNP distance between sampled hosts in red on edges.
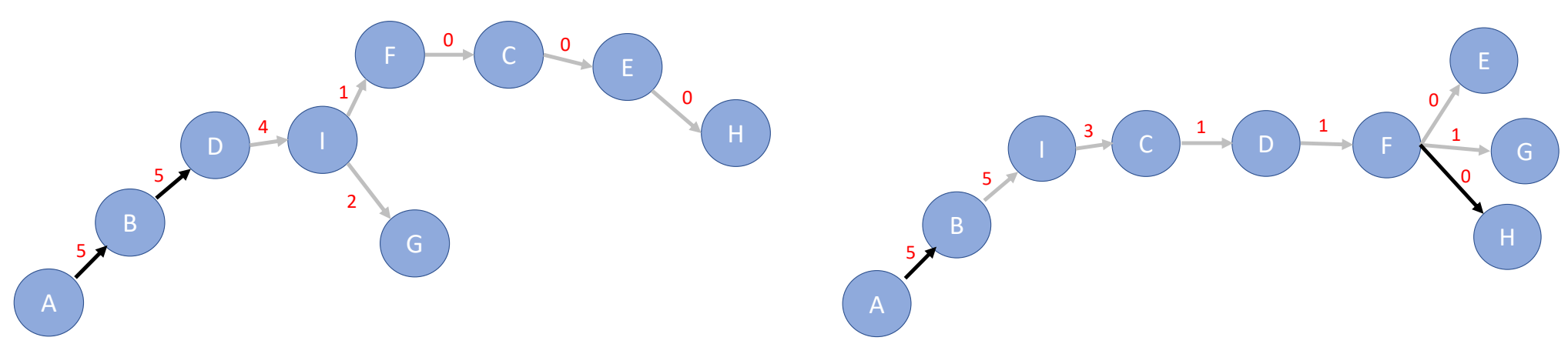
Table 2. The positive predictive value (PPV) and sensitivity of transmission pairs and epidemiologically linked pairs identified in clinical Mtb isolates from British Columbia.

\begin{tabular}{|l|c|c|c|c|c|c|}
\hline \multirow{2}{*}{$\begin{array}{l}\text { Transmission } \\
\text { reconstruction } \\
\text { model }\end{array}$} & \multicolumn{2}{|c|}{ All clusters } & \multicolumn{2}{c|}{ Small Clusters (N < 10) } & \multicolumn{2}{c|}{ Large Clusters (N $\geq 10)$} \\
\cline { 2 - 7 } & PPV & Sensitivity & PPV & Sensitivity & PPV & Sensitivity \\
\hline BEASTLIER ${ }^{19}$ & $15 / 320(5 \%)$ & $15 / 120(13 \%)$ & $5 / 25(20 \%)$ & $5 / 14(38 \%)$ & $10 / 295(3 \%)$ & $10 / 106(9 \%)$ \\
\hline Outbreaker2 ${ }^{26}$ & $14 / 133(11 \%)$ & $14 / 120(12 \%)$ & $1 / 10(10 \%)$ & $1 / 14(7 \%)$ & $13 / 123(11 \%)$ & $13 / 106(12 \%)$ \\
\hline Phybreak ${ }^{20}$ & $17 / 143(12 \%)$ & $17 / 120(14 \%)$ & $1 / 9(11 \%)$ & $1 / 14(7 \%)$ & $16 / 134(12 \%)$ & $16 / 106(15 \%)$ \\
\hline SCOTTI ${ }^{27}$ & $1 / 4(25 \%)$ & $1 / 120(1 \%)$ & $0 / 0(0 \%)$ & $0 / 14(0 \%)$ & $1 / 4(25 \%)$ & $1 / 106(1 \%)$ \\
\hline seqTrack ${ }^{24}$ & $8 / 80(10 \%)$ & $8 / 120(7 \%)$ & $1 / 4(25 \%)$ & $1 / 14(7 \%)$ & $7 / 76(9 \%)$ & $7 / 106(7 \%)$ \\
\hline TransPhylo ${ }^{16}$ & $20 / 135(15 \%)$ & $\mathbf{2 0 / 1 2 0 ( 1 7 \% )}$ & $5 / 14(36 \%)$ & $5 / 14(38 \%)$ & $15 / 119(13 \%)$ & $15 / 106(14 \%)$ \\
\hline TransPhyloMT ${ }^{25}$ & $\mathbf{1 8 / 6 3 ( 2 9 \% )}$ & $18 / 120(15 \%)$ & $\mathbf{6 / 1 1}(\mathbf{5 5} \%)$ & $\mathbf{6 / 1 4}(\mathbf{4 3} \%)$ & $\mathbf{1 2 / 5 2}(\mathbf{2 3} \%)$ & $12 / 106(11 \%)$ \\
\hline
\end{tabular}


Figure 3. Boxplots of the transmission parameters estimated by each tested method from high probability $(P \geq 0.5)$ transmission events between sampled clinical Mtb isolates from BC. (A) the SNP distance between observed hosts, and (B) the transmission interval between infection times of observed hosts.

A

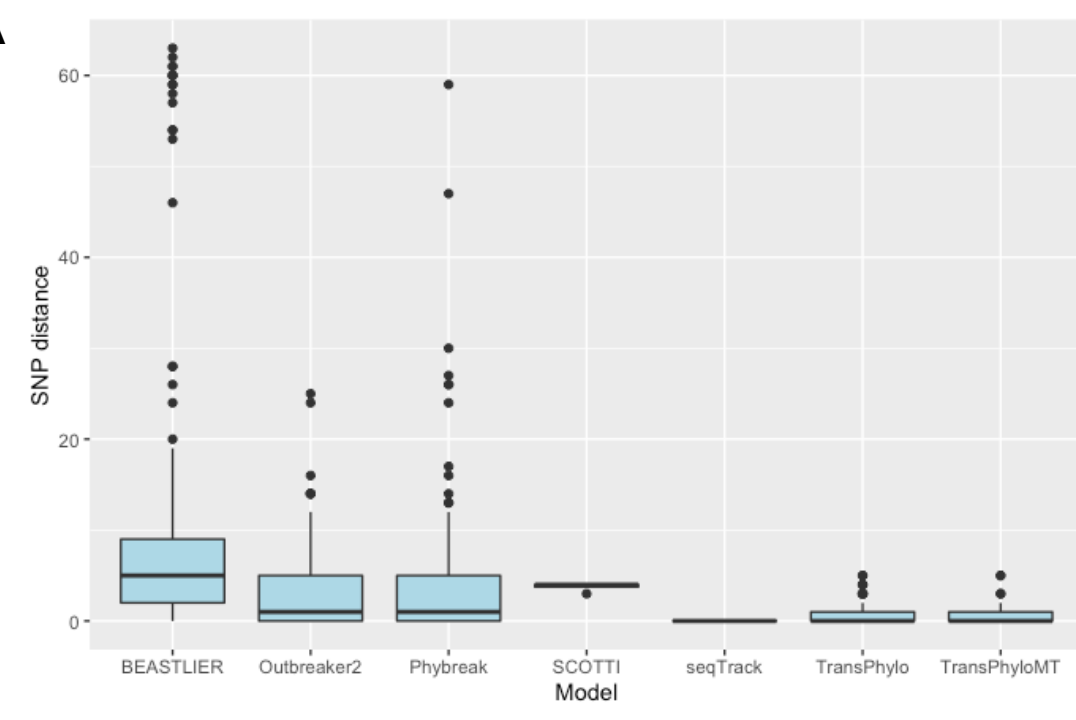

B

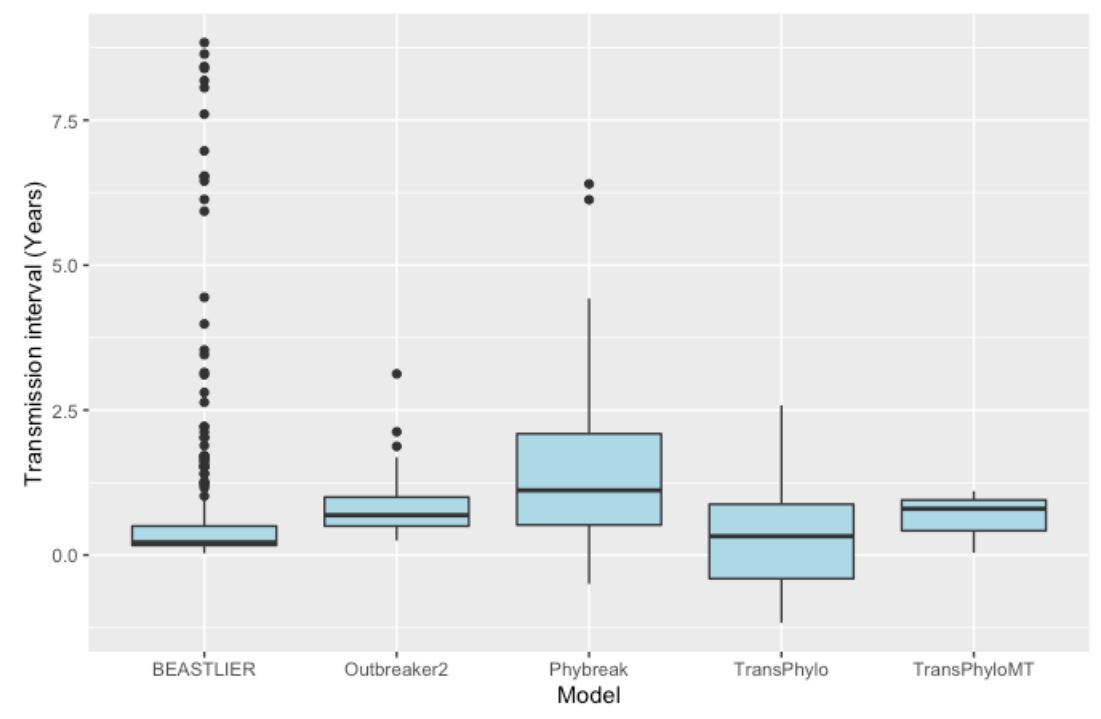

